\title{
Gestational Trophoblastic Neoplasia: Clinical and Therapeutic Profile in Madagascar
}

\author{
Norosoa Randriamaroson ${ }^{*}$, Malala Razakanaivo1, Ny Ony Andrianandrasana ${ }^{2}$, \\ Zo Ambinintsoa Solofonirina ${ }^{3}$, Florine Rafaramino ${ }^{3}$ \\ ${ }^{1}$ Radiotherapy Department, University Hospital Joseph Ravoahangy Andrianavalona, Antananarivo, Madagascar \\ ${ }^{2}$ Medical Oncology Department, University Hospital Joseph Ravoahangy Andrianavalona, Antananarivo, Madagascar \\ ${ }^{3}$ Faculty of Medicine, Antananarivo, Madagascar \\ Email: *randriamarosonnorosoa@gmail.com
}

How to cite this paper: Randriamaroson, N., Razakanaivo, M., Andrianandrasana, N.O., Solofonirina, Z.A. and Rafaramino, F. (2020) Gestational Trophoblastic Neoplasia: Clinical and Therapeutic Profile in Madagascar. Open Journal of Obstetrics and $G y$ necology, 10, 946-956.

https://doi.org/10.4236/ojog.2020.1070089

Received: May 28, 2020

Accepted: July 21, 2020

Published: July 24, 2020

Copyright $\odot 2020$ by author(s) and Scientific Research Publishing Inc. This work is licensed under the Creative Commons Attribution International License (CC BY 4.0).

http://creativecommons.org/licenses/by/4.0/

\begin{abstract}
Gestational trophoblastic disease (GTD) develops from abnormal cellular proliferation of trophoblasts following fertilization. This includes complete and partial hydatidiform mole (HM) and gestational trophoblastic neoplasia (GTN). The aim of this study was to report the epidemiological, clinical and therapeutic profile of gestational trophoblastic neoplasia (GTN) over period of ten years in the department of Oncology Radiotherapy at the University Hospital Joseph Ravoahangy Andrianavalona (HJRA) Antananarivo Madagascar. Medical records of women diagnosed with GTD in the department of Oncology Radiotherapy at HJRA from January 1st, 2007 to September 2017 were retrospectively reviewed. Only patients with the FIGO diagnosis GTN were included, while those with the histological diagnosis of hydatidiform mole (HM), also sometimes classified as GTD, were not included in this study. Also excluded were all cases with incomplete or missing data. Twenty four patients were included. Median age of patients at the time of diagnosis was 37 years (range 18 - 60). Most patients developed GTN following molar pregnancy (75\%), had disease duration from antecedent pregnancy of less than 6 months (58.20\%), and had the pre-treatment hCG level more than 10,000 IU/L (58.27\%). At diagnosis, 14 patients (58.33\%) had localized disease (M0). Most common metastatic sites at initial diagnosis were the liver and brain (20.83\%). After a median follow-up from initial diagnosis of six months (range 1 - 24), 58.33\% were lost to follow up. This represented an increase in the percentage of patients lost to follow up prior to completion of therapy, when compared with our previous results for an earlier time period. GTN in Malagasy woman displays an aggressive clinic profile. Finding ways to increase treatment compliance provides the best way to minimize recurrences of this potentially deadly disease.
\end{abstract}




\section{Keywords}

Choriocarcinoma, Gestational Trophoblastic Disease, Gestational Trophoblastic Neoplasia, Madagascar

\section{Introduction}

Gestational trophoblastic disease (GTD) is derived from the placenta and develops from abnormal cellular proliferation of trophoblasts following fertilization. GTD includes complete and partial Hydatidiform Mole (HM) as well as Gestational Trophoblastic Neoplasia (GTN). The subcategory of GTN, in turn, includes persistent invasive mole, choriocarcinoma, placental site trophoblastic tumors, and epithelioid trophoblastic tumor [1]. Hydatidiform moles and choriocarcinoma arise from villous trophoblast and placental-site trophoblastic tumours from interstitial trophoblasts. Epithelioid trophoblastic tumour is a variant of placental-site trophoblastic tumour with similar clinical behaviour but distinctive hyalinization has been reported [1]. Molar pregnancies may develop persistent elevated serum Human Chorionic Gonadotropin (HCG) levels after evacuation [1] [2] [3], with a chance of progression to choriocarcinoma (CCA). Most commonly, GTN is diagnosed following molar pregnancy. Any form of GTN can metastasize, and the most common metastatic site is lung (80\%) followed by vagina (30\%), brain, and liver (10\%) [4]. GTN is highly sensitive to chemotherapy and most curable cancer with success rate exceeding 90\% [4]. It represents one of the only cancers for which single-agent chemotherapy is still in wider use. Diagnosis and treatment are based on the International Federation of Gynecology and Obstetrics (FIGO) anatomic staging and prognostic scoring index. It is divided in two groups: low and high risk. The low-risk group can be treated with single-agent chemotherapy resulting in the survival rate approaching 100\%. The high-risk group requires initial multi-agent chemotherapy with or without adjuvant radiation therapy and surgery to achieve a survival rate of $80 \%-90 \%$ [5]. The last Malagasy study about GTN was back in 2004. However, there have been significant improvements in the delivery of cancer care in Madagascar since then, and GTN is a curable disease when treated correctly. The authors' aim was to scrutinize the epidemiological, clinical and therapeutic profile of GTN over the past ten years.

\section{Materials and Methods}

The retrospective data review was conducted in the department of Oncology Radiotherapy at HJRA. Medical records of women diagnosed with GTD from January 1st, 2007 to September 2017 were reviewed. Of these cases, all patients with the FIGO diagnosis criteria of GTN were included. These criteria are summarized in (Table 1) [5]. Histological HM were not included, and all incomplete files were excluded. The clinical data about patient and disease characteristics, 
Table 1. Criteria for the diagnosis of gestational trophoblastic neoplasia GTN.

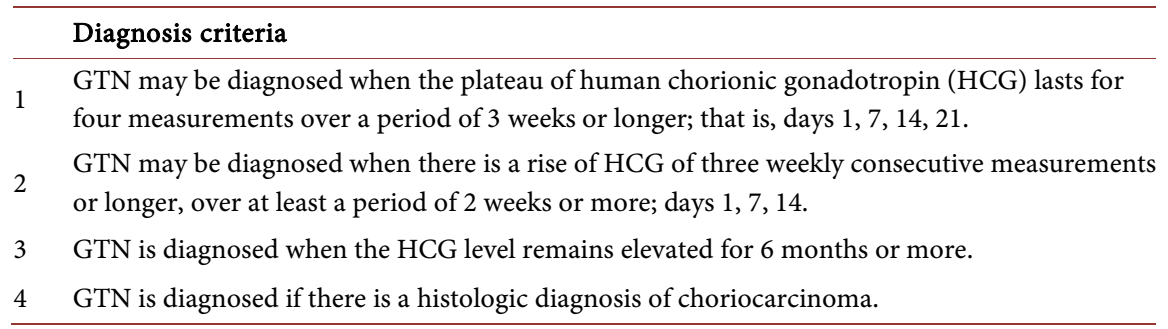

including age, pregnancy status, antecedent pregnancy, interval from pregnancy to diagnosis, pre therapeutic HCG level, means of diagnosis, clinico-pathologic type, tumor size, tumor stage, presence of metastatic disease, number of metastases, FIGO stage, modality of treatment, and follow up were recorded. The data thus collected was entered and analyzed by using EXCEL 2007. The anonymity and confidentiality of the data were strictly observed. This study has been approved by the local ethics committee.

\section{Results}

Twenty four patients were included. Patients and disease characteristics are presented in (Table 2). Median age of patients at the time of diagnosis was 37 years (range 18 - 60). Most patients developed GTN following molar pregnancy (75\%), had disease duration from antecedent pregnancy of less than 6 months (58.2\%), and had the pre-treatment HCG level more than 10,000 IU/L (58.27\%). Diagnosis was based on histopathologic evidence in four cases (16.7\%) and elevated hCG levels with history consistent with GTN in 20 (83.3\%) patients. GTN occurred after full-term pregnancy in two cases (8.33\%). At diagnosis, 14 patients (58.33\%) had localized disease (M0), whereas ten (41.67\%) had metastasis. Most common metastatic sites at initial diagnosis were the liver and brain (20.83\%) spleen and kidney metastasis (12.5\%), lung metastasis (8.33\%). Number of metastases is reported in Figure 1. Based upon the staging work-up, patients with GTN were categorized according to the FIGO anatomic staging and the World Health Organization (WHO) scoring system called the prognostic scoring index. The WHO risk factor scoring system was determined by age, antecedent pregnancy, interval from antecedent pregnancy, pre-treatment HCG level, largest tumor size, site of metastasis, number of metastasis, and previous failed chemotherapy. Patients with score $<7$ were considered low-risk, whereas patients with score 7 or more were considered high-risk group [5]. Four patients (16.60\%) stage I, seven patients (29.17\%) stage II, five patients (20.83\%) stage III and eight patients $(33.33 \%)$ stage IV. With regards to the scoring system, $11(45.83 \%)$ cases were scored low risk and 13 (54.17\%) high risk. In the low-risk group, ten patients received single agent methotrexate (MTX). In high-risk group, nine patients received combination chemotherapy methotrexate and cyclophosphamide. We noted that five patients did not receive chemotherapy. Two patients underwent preventive hysterectomy for excessive uterine bleeding: one in the low risk group and one in the high risk group. No patient received radiation therapy treatment. 
Table 2. Patients and disease characteristics.

\begin{tabular}{|c|c|}
\hline Demographics & Number $=24$ \\
\hline Median age (range) & 37 years $(18-60)$ \\
\hline$<40$ years & $13(54.17 \%)$ \\
\hline$\geq 40$ years & $11(45.83 \%)$ \\
\hline \multicolumn{2}{|l|}{ Pregnancy status } \\
\hline Median & 5 \\
\hline$<4$ & $7(29.16 \%)$ \\
\hline$\geq 4$ & $17(70.84 \%)$ \\
\hline \multicolumn{2}{|l|}{ Antecedent pregnancy } \\
\hline Hydatidiform mole & $18(75.00 \%)$ \\
\hline Abortion & $4(16.67 \%)$ \\
\hline Term pregnancy & $2(8.33 \%)$ \\
\hline \multicolumn{2}{|l|}{ Interval } \\
\hline$<6$ months & $14(58.20 \%)$ \\
\hline $7-12$ months & $2(8.33 \%)$ \\
\hline$>12$ months & $5(20.83 \%)$ \\
\hline Unknown & $3(12.50 \%)$ \\
\hline \multicolumn{2}{|l|}{ Pre-treatment HCG level } \\
\hline$<1000$ & $6(25.00 \%)$ \\
\hline $1000-<10,000$ & $4(16.67 \%)$ \\
\hline $10,000-<100,000$ & $7(29.17 \%)$ \\
\hline$\geq 100,000$ & $7(29.10 \%)$ \\
\hline \multicolumn{2}{|l|}{ Clinico-pathologic type } \\
\hline Persistent/invasive GTN & $5(20.83 \%)$ \\
\hline Choriocarcinoma & $19(79.17 \%)$ \\
\hline \multicolumn{2}{|l|}{ Tumor size } \\
\hline$<4$ centimeters $(\mathrm{cm})$ & $8(33.33 \%)$ \\
\hline$>5$ centimeters $(\mathrm{cm})$ & $8(33.33 \%)$ \\
\hline Unknown & $8(33.33 \%)$ \\
\hline \multicolumn{2}{|l|}{ FIGO stage } \\
\hline I & $4(16.60 \%)$ \\
\hline II & $7(29.17 \%)$ \\
\hline III & $5(20.83 \%)$ \\
\hline IV & $8(33.33 \%)$ \\
\hline \multicolumn{2}{|l|}{ Radiation therapy } \\
\hline No & $24(100 \%)$ \\
\hline \multicolumn{2}{|l|}{ Surgery } \\
\hline Uterine evacuation & $22(91.66 \%)$ \\
\hline Hysterectomy & $2(8.33 \%)$ \\
\hline Metastasectomy & $0(0.00 \%)$ \\
\hline \multicolumn{2}{|l|}{ Chemotherapy } \\
\hline Single agent Methotrexate & $10(41.66 \%)$ \\
\hline Combination chemotherapy & $9(37.50 \%)$ \\
\hline No chemotherapy & $5(20.83 \%)$ \\
\hline
\end{tabular}




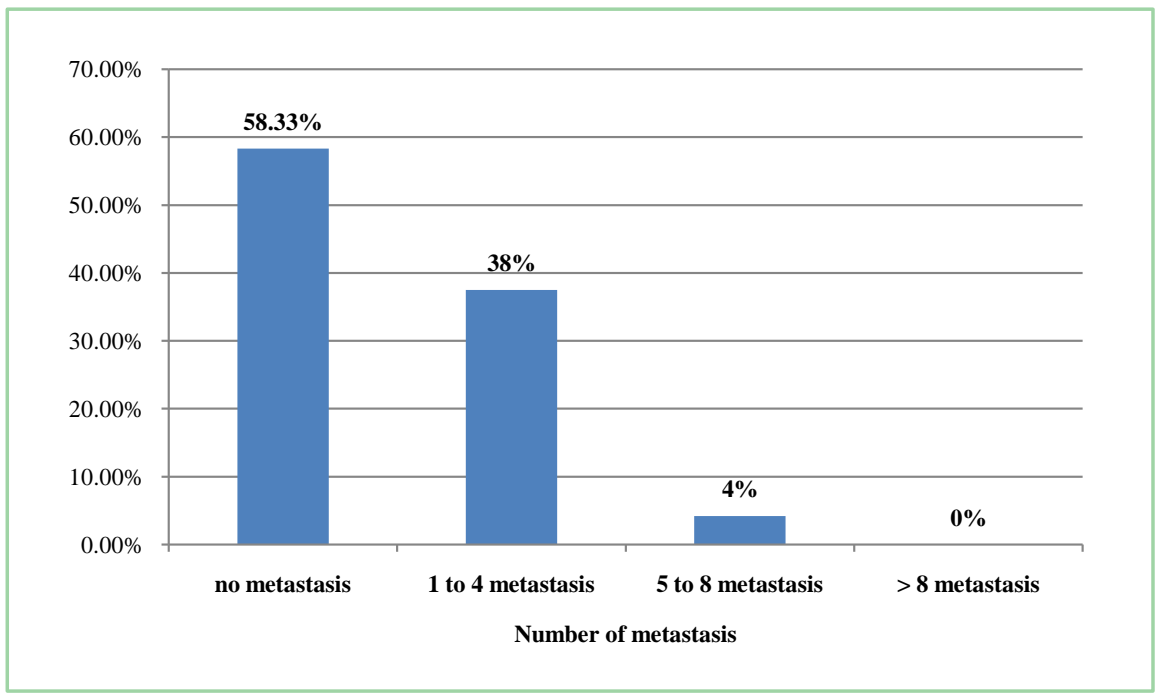

Figure 1. Number of metastasis.

After treatment of GTN, frequent monitoring of HCG for at least 12 months with reliable contraception is essential for surveillance of relapse. The response to treatment was evaluated during follow-up by clinical examination, HCG levels and imaging as and when required. After a median follow-up from initial diagnosis of six months (range 1 - 24), 58.33\% were lost, before achieving complete response, $25 \%$ achieved complete response (three in stage I, three in stage IV), $16.66 \%$ died (all in stage IV, two due to disease progression and two to chemotherapy toxicity).

\section{Discussion}

GTN can arise after any type of pregnancy and can develop months or years after prior pregnancy. It affects women in the age group of 20 to 30 years [6]. Rim Batti et al. reported 109 patients presented with GTN with an average age of 34 years [7]. Tsuji $\mathrm{K}$ reported that $37 \%$ of women older than 40 developed post-molar GTN as compared to a lower $15 \%-20 \%$ in young patients. These results suggest that hydatidiform mole may have a greater potential for malignant transformation in patients 40 years and older than in younger patients [8]. In our study, the median age was 37 years, $45.85 \%$ of our patients are older than 40 years old, which comparable to that reported in the literature. Therefore, it can be deduced that maternal age is proportionately associated with post-molar GTN, and the incidence of GTN is significantly higher when patients reach the age of 40 and older, based on which we defined 40 years old as the cut-off value.

Approximately 50\% of all cases of GTN occur after molar gestations, 25\% after abortions or ectopic pregnancies and $25 \%$ after term or preterm deliveries [9]. Two studies, by Golfier and Bjorje, have reported 79\% and 75\% GTN after molar gestations respectively, which is in comparison with our study [10] [11]. Most GTN occur after molar gestations but it should be noted that they can also occur after other types of gestation. Approximately $15 \%$ of all molar pregnancies 
will develop into GTN [12] [13]. The National Comprehensive Cancer Network (NCCN) Guidelines use the FIGO staging criteria for post molar GTN as meeting one of more criteria after treatment of HM, as indicated by hCG monitoring. After HM, this HCG surveillance is important to avoid a delayed diagnosis in GTN because post molar GTN is typically diagnosed using HCG surveillance. Delayed diagnosis of postpartum CCA may increase the risk of metastasis and mono chemotherapy resistance, thus affecting its prognosis [14]. Dhanda $\mathrm{S}$ reported that most patients developed invasive mole (IM) within 6 months after previous pregnancy, especially within two to three months. On the other hand, CCA may present several years after antecedent pregnancy [15].

In our study, 58.20\% developed GTN within six months after previous pregnancy. Yet late presentations and late diagnoses were also seen in a portion of the patient population. Even though more than fifty percent of GTN were diagnosed within 6 months after previous pregnancy, 20.83\% were diagnosed after 12 months. We cannot assess whether these patients followed the HCG surveillance as indicated or not. Our findings are consistent with the results reported in literature and indicate the importance of standard follow-up for the patients with GTN displaying abnormal rise of hCG and complications [16]. Markedly elevated HCG values are suggestive of diagnosis of GTN as well as an index for diagnosis of recurrence and curative effect [17]. Batti in his study reported a mean pre-treatment hCG level of 152,170 UI/l (median 10,068) [7]. The concentration of hCG before treatment in this study varied considerably. We found $58.27 \%$ with hCG level more than $10,000 \mathrm{U} / \mathrm{ml}$, which is the one poor prognostic factor as previously reported [18]. It is possible that GTN in Malagasy people are aggressive with more than fifty percent diagnosed within 6 months after previous pregnancy but diagnosed with a high level HCG. Batti and al husaini reported respectively in their study $57.14 \%$ and $41 \%$ in stage I [7] [19]. Compared with our results, most patients with GTN were diagnosed at later stages, with locally advanced and metastatic disease, and with just $16.60 \%$ in stage I. These results showed an aggressive clinic profile in our patients. Regarding metastases of GTN, our data showed that the most common metastatic sites at initial diagnosis were liver and brain. This is quite different from prior investigations where GTN metastasis were lungs and vulvo vaginal region, followed by the brain and liver [7] [20]. Prevalence of liver metastasis from GTN is approximately $1.8 \%-3.4 \%$ [21]. Patients with liver and/or brain metastasis have poorer prognosis [21]. As with brain metastasis, liver metastases are rare and more likely to manifest with non molar GTN and in the presence of other distant metastases [22]. IM and CCA, which make up the vast majority of GTN, were the two types reported in this study. These always substantial amounts of HCG and are highly responsive to chemotherapy with an overall cure rate exceeding $90 \%$, making it usually possible to achieve cure while preserving fertility [12]. This success is due to the unique sensitivity of these two trophoblastic neoplasms to chemotherapy and the use of HCG as a tumor marker for diagnosis, monitoring treatment and follow-up [23]. 
Patients with low-risk GTN (WHO risk score 0-4) should be treated single agent methotrexate or actinomycin D. Numerous studies have evaluated these agents, but differences in inclusion criteria and dosages have made it challenging to determine a superior regimen. Although some experts consider methotrexate to have more favorable adverse effect profile, dactinomycin may achieve similar or better efficacy with a less-frequent infusion schedule [24]. The Cochrane Review in 2012, including 513 patients in five randomized controlled trials, showed that actinomycin D (Act-D) appeared to be superior to methotrexate (MTX) (risk ratio [RR] 0.64; 95\% confidence interval, [CI] 0.54 - 0.76) [25]. As first-line chemotherapy, pulse Act-D was effective and tolerable for patients with low-risk post-molar non-choriocarcinoma. Existing invasive uterine lesions observed by pre-chemotherapy transvaginal ultrasound, a FIGO score $\geq 5$, and pre-chemotherapy levels of HCG $\geq 4000$ IU/L were independent factors for resistance to pulse Act-D [26]. A 2016 Cochrane Database review of restricted studies to RCTs in low-risk GTN showed with moderate-certainty evidence that first-line methotrexate may be more likely to fail than dactinomycin (RR, 3.55; 95\% CI, 1.81 - 6.95; 6 trials, 577 participants; I(2)561\%) [25]. Similarly, the authors concluded that dactinomycin is more likely to lead to a primary cure than methotrexate (RR, $0.65 ; 95 \%$ CI, 0.57 - 0.75; 6 trials, 577 participants; I(2)526\%); however, 55\% of the data came from trials of weekly intramuscular methotrexate, which seems to be less effective than the 5- or 8-day methotrexate regimens [25]. As said in these review, GTN is a curable disease by using Chemotherapy. It is not sufficient to treat patient but it requires an HCG dosage as a tumor marker during treatment. The cost of treatment and this marker tumor in each cure are expensive for a low income country like Madagascar. They can afford the treatment in the beginning but when they spent all of these money, they dropped the treatment. Due to decreased cost and the good tolerance of Methotrexate, especially for not inducing hair loss, Methotrexate was preferred as firstline treatment in this study. However a recent study suggest that women with hCG $>400,000 \mathrm{UI} / 1$ are unlikely to be cured by single agent chemotherapy and should be treated by multi-agent chemotherapy as first line treatment [27]. High-risk GTN should be treated with multiagent chemotherapy. Adjuvant surgery or radiation therapy may be included. With a multimodal approach, cure rates have reached approximately $90 \%$, including almost all patients with only lung/vaginal metastases and 70\% for patients with stage IV disease [28]. We treated High risk GTN in this study with multiagent chemotherapy alone without adjuvant surgery or radiation therapy even if there were liver and brain metastasis most reported. Ahmed et al. reported that factors associated with poorer outcomes include liver and brain metastases, particularly if co-occurring; however, the prognosis for these patients has improved over time [21]. Rahantarison in 2004 reported that $34 \%$ underwent first line hysterectomy [29]. Surgery may have an important role in the management of GTN. Hysterectomy can be considered in uncontrolled uterine bleeding, although it can often be avoided with the use of uterine artery embolization. 
Laparotomy may be needed to stop bleeding in organs such as the liver, gastrointestinal tract, kidneys, and spleen. Neurosurgery is needed if there is bleeding into the brain or increased intracranial pressure. The resection of an isolated drug-resistant tumor may also be curative [30]. Hary Fanambinantsoa Rabarikoto et al. reported a laparotomy in emergency which discovered an important bleeding from the fundus of the uterus which has been perforated, leading up to realize an emergency hemostasis hysterectomy [31]. In our study, patients with uncontrolled uterine bleeding $8.33 \%$ underwent hysterectomy. There were no difficulty to reach this emergency surgery in Madagascar because patients can pay the surgery cost after beeing treated even if they cannot afford it. Most of Hospitals in Madagascar offer this opportunity for those who did not have money for their treatment but the patients cannot leave the Hospital before paying their debt. Most of the time, they find a solution to solve this surgery problem, but after, they forsaken the next step of the treatment such as chemotherapy with HCG tumor marker in each cure. Regarding radiotherapy, it has a limited role in GTN, except in treatment of brain metastasis, although it efficacy compared with intrathecal methotrexate is controversial [30]. One patient with brain metastasis underwent Radiotherapy in the last study in this topic reported by Rahantarison in 2004 [29]. During this study period, the Radiotherapy center in Madagascar was broken, and for this reason that patient with brain metastasis did not undergo Radiotherapy. More than fifty percent (58.33\%) of the patients in this study were lost to follow up and $25 \%$ achieved complete response. While the previous study by Rahantarison in 2004 reported a much higher complete remission compared to that found in our study, this complete remission was $42.5 \%$ in patients with a follow-up range to 1 to 30 months [29]. Our result is accounted for by the high proportion of patients lost to follow up. We cannot conclude and find the therapeutic aspect of GTN with a lot of lost to follow up. Finding all the reasons which make them not fulfil their treatment until the end is the best way to avoid recurrence and improve the results of Malagasy GTN treatment in the future.

\section{Limitations}

The limits of the study are those of a retrospective study with missing or incomplete data and, at times, insufficient detail of recorded data for treatment and follow up.

\section{Conclusion}

GTN appears most often after post molar pregnancy. Choriocarcinoma remains the most represented after a diagnostic delay similar to that reported in the literature with an aggressive clinic profile. Most common metastatic sites at initial diagnosis were the liver and brain. Compared to the last study, before having complete response, the number of patients who lost follow-up has been increased. It is important to further investigate reasons for this increased loss to follow up and decreased treatment compliance in Malagasy patients with GTN. 


\section{Acknowledgements}

The authors would like to gratefully thank all the staff of the Department of Oncology Radiotherapy at the University Hospital Joseph Ravoahangy Andrianavalona (HJRA) Antananarivo Madagascar for the administrative authorization. They are deeply indebted to the patient who by his consent allows the caregivers to improve their skills in providing care to patients with gestational trophoblastic disease.

\section{Conflicts of Interest}

The authors declare no conflicts of interest regarding the publication of this paper.

\section{References}

[1] Seckl, M.J., Sebire, N.J. and Berkowitz, R.S. (2010) Gestational Trophoblastic Disease. Lancet, 376, 717-729. https://doi.org/10.1016/S0140-6736(10)60280-2

[2] Barry, W., Hancock, M.D., Kauzer Nazir, M.D., Janet, E. and Everard, S.R.N. (2006) Persistent Gestational Trophoblastic Neoplasia after Hydatiform Mole Incidence and Outcome. Journal of Reproductive Medicine, 51, 764-766.

[3] Laurain, J.R. (2010) Gestational Trophoblastic Disease: Epidemioogy, Pahology, Clinical Presentation and Diagnosis of Gestational Trophoblastic Disease, and Management of Hydatiform Mole. American Journal of Obstetrics and Gynecology, 203, 531-539. https://doi.org/10.1016/j.ajog.2010.06.073

[4] Soper, J.T. (2006) Gestational Trophoblastic Disease. Obstetrics \& Gynecology, 108, 176-187. https://doi.org/10.1097/01.AOG.0000224697.31138.a1

[5] Ngan, H.Y., Bender, H., Benedet, J.L., Jones, H., Montruccoli, G.C. and Pecorelli, S. (2003) Gestational Trophoblastic Neoplasia, FIGO 2000 Staging and Classification. International Journal of Gynecology \& Obstetrics, 83, 175-177. https://doi.org/10.1016/S0020-7292(03)90120-2

[6] Sita-Lumsden, A., Short, D., Lindsay, I., Sebire, N.J., Adjogatse, D., Seckl, M.J. and Savage, P.M. (2012) Treatment Outcomes for 618 Women with Gestational Trophoblastic Tumours Following a Molar Pregnancy at the Charing cross Hospital, 2000-2009. British Journal of Cancer, 107, 1810-1814. https://doi.org/10.1038/bjc.2012.462

[7] Rim, B., Amina, M., et al. (2019) Gestational Trophoblastic Neoplasia: Experience at Salah Azaiez Institute. Pan African Medical Journal, 33, 121-120.

https://doi.org/10.11604/pamj.2019.33.121.13897

[8] Tsuji, K., Yagi, S. and Nakono, R. (1981) Increased Risk of Malignant Transformation of Hydatidiform Moles in Older Gravidas: A Cytogenetic Study. Obstetrics \& Gynecology, 58, 35-355.

[9] Goldstein, D.P. and Berkowitz, RS. (2012) Current Management of Gestational Trophoblastic Neoplasia. Hematology/ Oncology Clinics of North America, 26, 111-131. https://doi.org/10.1016/j.hoc.2011.10.007

[10] Golfier, F., Labrousse, C., et al. (2007) Evaluation de la Prise en Charge des Tumeurs Trophoblastiques Gestationnelles Enregistrées au Centre de Référence des Maladies Trophoblastiques de Lyon de 1999 à 2005. Gynécologie Obstétrique \& Fertilité, 35, 205-215. https://doi.org/10.1016/j.gyobfe.2006.12.023 
[11] Bjorje, T., and Abeler, V.M., Sundfør, K., Tropé, C.G. and Kærn, J. (1987) Gestational Trophoblastic Tumors in Norway, 1968-1997: Patient Characteristics, Treatment and Prognosis. Gynecologic Oncology, 87, 71-76.

https://doi.org/10.1006/gyno.2002.6801

[12] Berkowitz, R.S. and Goldstein, D.P. (2009) Current Management of Gestational Trophoblastic Diseases. Gynecologic Oncology, 112, 654-662. https://doi.org/10.1016/j.ygyno.2008.09.005

[13] Goto, S., Yamada, A., Ishizuka, T. and Tomoda, Y. (1993) Development of Postmolar Trophoblastic Disease after Partial Molar Pregnancy. Gynecologic Oncology, 48, 165-170. https://doi.org/10.1006/gyno.1993.1028

[14] Lage, J.M., Gompel, C. and Silverberg, S.G. (1994) The Placenta. Pathology in Obstetrics and Gynecology. 4th Edition, Lippincott Williams and Wilkins, Philadelphia, PA, 448.

[15] Dhanda, S., Ramani, S. and Thakur, M. (2014) Gestational Trophoblastic Disease: A Multimodality Imaging Approach with Impact on Diagnosis and Management. Radiology Research and Practice, 2014, Article ID: 842751. https://doi.org/10.1155/2014/842751

[16] Garner, E.I.O., Goldstein, D.P., et al. (2007) Gestational Trophoblastic Disease. Clinical Obstetrics and Gynecology, 50, 112-122. https://doi.org/10.1097/GRF.0b013e31802f17fc

[17] Wang, S., An, R.F., Han, X.B., Zhu, K.X. and Xue, Y. (2006) Combination Chemotherapy with 5-Fluorouracil, Methotrexate and Etoposide for Patients with High-Risk Gestational Trophoblastic Tumors: A Report Based on Our 11-Year Clinical Experiences. Gynecologic Oncology, 103, 1105-1108. https://doi.org/10.1016/j.ygyno.2006.06.031

[18] Niemann, I., Petersen, L.K., et al. (2006) Predictors of Low Risk of Persistent Trophoblastic Disease in Molar Pregnancies. Obstetrics \& Gynecology, 107, 1006-1011. https://doi.org/10.1097/01.AOG.0000210635.24543.3b

[19] Al-Husaini, H., Soudy, A., et al. (2014) Gestational Trophoblastic Neoplasia: Treatment Outcomes from a Single Institutional Experience. Clinical and Translational Oncology, 17, 409-415. https://doi.org/10.1007/s12094-014-1251-1

[20] Milenković, V., Lazović, B., Mačvanski, M., Jeremić, K. and Hrgović, Z. (2013) Clinical Outcome of a FIGO Stage IV Gestational Choriocarcinoma. Case Reports in Oncology, 6, 504-507. https://doi.org/10.1159/000353626

[21] Ahamed, E., Short, D., Bernard, N., Savage, P.M. and Seckl, M.J. (2012) Survival of Women with Gestational Trophoblastic Neoplasia and Liver Metastases: Is It Improving? The Journal of Reproductive Medicine, 57, 262-269.

[22] Barber, E.L., Schink, J.C. and Lurain, J.R. (2014) Hepatic Metastasis in Gestational Trophoblastic Neoplasia: Patient Characteristics, Prognostic Factors, and Outcomes. The Journal of Reproductive Medicine, 59, 199-203.

[23] Sung, W.J., Shin, H.C., Kim, M.-K. and Kim, M.J. (2013) Epithelioid Trophoblastic Tumor: Clinicopathologic and Immunohistochemical Analysis of Three Cases. Journal of Pathology and Translational Medicine, 47, 67-73. https://doi.org/10.4132/KoreanJPathol.2013.47.1.67

[24] Nadeem, R., Abu-Rustum, M.D., et al. (2019) Gestational Trophoblastic Neoplasia. Journal of the National Comprehensive Cancer Network, 17, 1374-1391.

[25] Lawrie, T.A., Alazzam, M., Tidy, J., Hancock, B.W. and Osborne, R. (2016) First-Line Chemotherapy in Low-Risk Gestational Trophoblastic Neoplasia. Cochrane Database Systematic Reviews, No. 6, Article No. CD007102. https://doi.org/10.1002/14651858.CD007102.pub4 
[26] Li. L., Wan, X.R., et al. (2018) Pulse Actinomycin D as First-Line Treatment of Low-Risk Post-Molar Non-Choriocarcinoma Gestational Trophoblastic Neoplasia. BMC Cancer, 18, Article No. 585. https://doi.org/10.1186/s12885-018-4512-5

[27] Mcgrath, S., Short, D., Harvey, R., Schmid, P., Savage, P.M. and Seckl, M.J. (2010) The Management and Outcome of Women with Post-Hydatidiform Mole "Low-Risk" Gestational Trophoblastic Neoplasia, but hCG Levels in Excess of $100000 \mathrm{IU}^{-1}$. British Journal of Cancer, 102, 810-814. https://doi.org/10.1038/sj.bjc.6605529

[28] Lurain, J.R. (2011) Gestational Trophoblastic Disease II: Classification and Management of Gestational Trophoblastic Neoplasia. American Journal of Obstetrics \& Gynecology, 204, 11-18. https://doi.org/10.1016/j.ajog.2010.06.072

[29] Rahantarison, C. (2004) Les Tumeurs Trophoblastiques Gestationnelles. Doctorat en Médecine. Université d'Antananarivo Faculté de Médecine, Antananarivo.

[30] Ngan, H.Y., Kohorn, E.I., et al. (2012) Trophoblastic Disease. International Journal of Gynecology \& Obstetrics, 119, S130-S136. https://doi.org/10.1016/S0020-7292(12)60026-5

[31] Rabarikoto, H.F., Hasiniatsy, N.E.R., Razafindrafara, H.E. and Randriambololona, D.M.A. (2018) Invasive Mole Complicating a Spontaneous Abortion. ARC Journal of Gynecology and Obstetrics, 3, 21-24. 